\title{
Steel Structures: Brilliant Ideas, Advances and Discussions
}

\section{Ghanbari Ghazijahani T*}

School of Engineering and ICT, University of Tasmania, Hobart, Australia

\section{General Outlook}

Brilliant ideas are worth as much as a complete research. Steel structures have followed a quite well-trodden and yet challenging path, particularly with the advent of new materials in construction industries. As a result, innovative and stimulating ideas played a significant part, since both researchers involving in constructions and/or industries drew comparisons among different materials to achieve most optimal ideas. In different research and construction projects, the major concern that whether sole material or a composition of two (or more) materials outweigh, has always been raised among decision makers. Notwithstanding, the significant role of steel as a crucial element in construction has remained inarguable. Despite this, the need for new ideas has been always vitally felt to come up with innovations in steel comparable with concrete and other materials. To this end, Journal of Steel Structure and Construction (JSSC) aims to provide an open international forum for bright ideas on steel structures. This paper outlines the significance of the ideas in steel elements and puts few instances forward among the new advances.

\section{Interesting Ideas and JSSC's Inclinations}

It is believed that creative ideas in steel structures and in turn construction can indeed provide the readers with remarkable insights leading their topics to reasonable directions. In this journal - as its inherent responsibility in dealing with researches on steel elements has recently started - no limit is placed on the type of the innovations for consideration for publication, i.e. if the ideas are expressed in an organized fashion with sufficient contribution to the filed, they will be considered for the publication following a peer review. JSSC aspires to take short original notes on different aspects of steel structures into account for publication provided that steel as the major element is included. This, should not however, discourage researches on composite elements whatsoever, wherein steel is comprised in materials.

\section{Geometric Non-uniformities and Recent Advances}

Unlike other materials in civil engineering, steel structures are more vulnerable to the uniformity or perfection of the geometry. There have been some advances over the past few years about the uniformity of the geometry in which the normal fabrication-related imperfections or post-construction imperfections were evaluated against perfect structures. This section aims to point out a number of instances to stimulate authors of this field that the topics about the integrity of any structural element are welcome to be considered. In all references the author and the collaborators have tries to show the detrimental effects of non-uniformities [1-5], i.e, in terms of rate and criticality of the mentioned effects.

Unlike this, though, an effort was made [6-8] to set forth the positive effects of irregularities provided that they are designed in a professional ways, e.g. corrugations and/or stiffeners in thin-walled structures, all of which implied the strengthening effect of some particular imperfections on the body of such structures. It is noteworthy that material nonuniformities are also found to be more supportive to the idea of the positive effects of geometrical irregularities in the aforementioned references.

\section{Different Materials and Researchers Interests}

Although different materials have been put forward and accordingly developed in the construction industry, steel - as a conventional material in building and construction - has not lost its widespreadness among both researchers and people dealing with construction and performance. It is, however, fitting to mention that concrete among the other materials has competed well and therefore, remarkably caught eyes of different researchers over the past decades. Besides, carbon fiber reinforced plastic is vastly seen particularly within the last twenty years. Notwithstanding, steel as a single material in general - due to its inherent feature of being light weight and yet strong - and in combination with the other materials in particular, still plays a key role in the construction industry. Highly cited papers, nowadays, are seen on the topics concerning with the rehabilitation of the steel with the new materials, which are also welcome to be considered in JSSC.

\section{Usage of Analytical Methods to Find the Critical Points in Steel Structures}

In recent years a number of researches have been conducted to identify the critical points of thin-walled steel structures. As examples, Ghanbari Ghazijahani and Zirakian performed a study to utilize the data obtained from the experiments on conical shells subjected to the external pressure in order to find the critical buckling load by extrapolation techniques. This method has been established first for steel beams and columns and was applied to the shell structures [9] (Figure 1). This set of data resulted in a very good agreement comparing experimental data with the results of the extrapolation methods. This reference as a single example is deemed to be developed to the variety of other usages especially when it comes to steel members with a relatively masked critical response, e.g. buckling loads. Further studies on steel structures are still required and encouraged in this regard to extend the knowledge on this topic (Figure 1).

\section{Summary}

This paper briefly outlines the significance of innovative ideas to be included in research topics of steel structures. To this end, a comparison was drawn between different existing materials and the role of steel was highlighted as a key element in composite members. The geometric irregularities of steel structures and their impact on the capacity was discussed and the new advances were included in this paper to motivated the readers to chase the mentioned topic. Analytical

*Corresponding author: Ghanbari Ghazijahani T, School of Engineering and ICT, University of Tasmania, Hobart, Australia, Tel: (+61) (0)469 311896 ; E-mail: tohid.ghanbari@utas.edu.au

Received August 25, 2015; Accepted August 26, 2015; Published September 03, 2015

Citation: Ghanbari Ghazijahani T (2015) Steel Structures: Brilliant Ideas, Advances and Discussions. J Steel Struct Constr 1: e101. doi:10.4172/2472-0437.1000e101

Copyright: (C) 2015 Ghanbari Ghazijahani T. This is an open-access article distributed under the terms of the Creative Commons Attribution License, which permits unrestricted use, distribution, and reproduction in any medium, provided the original author and source are credited. 

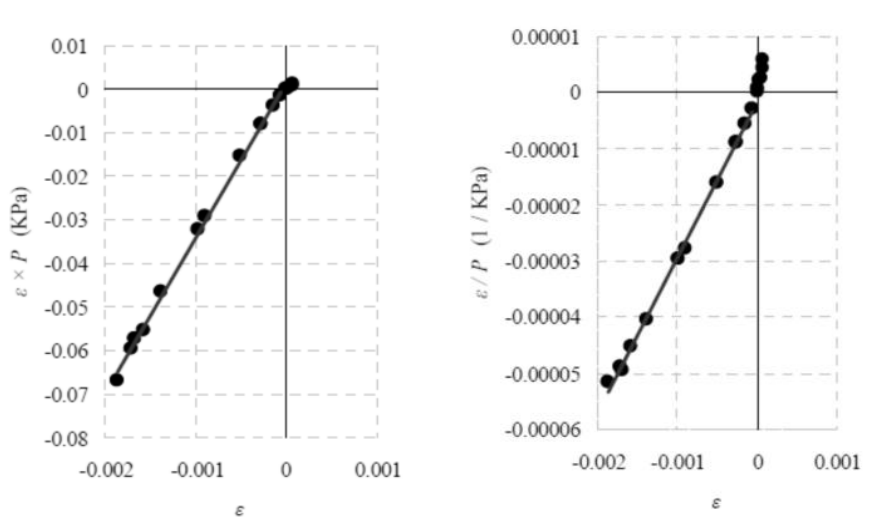

Figure 1: Typical Modified and Southwell Plots for steel shells [9].

methods in finding the critical points of the capacity for steel structures were also pointed out, which could be a good point of departure for the future relevant works.

\section{References}

1. Ghanbari Ghazijahani T, Jiao H, Holloway D (2014) Experimental study on damaged cylindrical shells under compression. Thin-Walled Structures 80: 13-21.

2. Ghanbari Ghazijahani T, Jiao H, Holloway D (2014) Experiments on dented cylindrical shells under peripheral pressure. Thin-Walled Structures 84: 50-58.

3. Ghanbari Ghazijahani T, Jiao H, Holloway D (2015) Fatigue tests of damaged tubes under flexural loading. Steel and Composite Structures 19: 223-236.

4. Ghanbari Ghazijahani T, Jiao H, Holloway D (2015) Plastic buckling of dented steel circular tubes under axial compression. Thin-Walled Structures 92: 48-54.

5. Ghanbari Ghazijahani T, Jiao H, Holloway D (2015) Holloway Fatigue experiments on circular hollow sectionswith CFRP reinforced cutouts. Journal of Constructional Steel Research106: 322-328.

6. Ghanbari Ghazijahani T, Jiao H, Holloway D (2015) Longitudinally stiffened corrugated cylindrical shells under uniform external pressure. Journal of Constructional Steel Research 110: 191-199.

7. Ghanbari Ghazijahani T, Dizaji HS, Nozohor J, Zirakian T (2015) Experiments on corrugated thin cylindrical shells under uniform external pressure. Ocean Engineering 106: 68-76.

8. Ghanbari Ghazijahani T, Jiao H, Holloway D (2014) An experimental study on externally pressurized stiffened and thickened cylindrical shells. Thin-Walled Structures 85: 359-366.

9. Ghanbari Ghazijahani T, Zirakian T (2014) Determination of buckling loads of conical shells using extrapolation techniques. Thin-Walled Structures 74: 292-299. 